\title{
Synthesis of reflections and conclusions of the SFRP-IRPA workshop on the reasonableness in the practical implementation of the ALARA principle
}

\author{
T. Schneider ${ }^{1}$, J.-F. Lecomte ${ }^{2}$, C. Schieber ${ }^{1}$, S. Andresz ${ }^{1}$, V. Chambrette ${ }^{3, *}$, \\ B. Le Guen ${ }^{4}$ and L. Vaillant ${ }^{1}$ \\ ${ }^{1}$ CEPN, 92260 Fontenay-aux-roses, France. \\ 2 IRSN, DAI, 92262 Fontenay-aux-roses, France. \\ 3 SFRP, 92263 Fontenay-aux-roses, France. \\ 4 EDF, IGSN, 75008 Paris, France.
}

Received: 4 July 2017 / Accepted: 25 September 2017

\begin{abstract}
For almost two years, the International Radiological Protection Association (IRPA) has been engaged in a reflection on the implementation of the radiological protection system and its evolution. In this context, the French Society for Radiological Protection (SFRP) organised a workshop on the search for a level of protection judged to be "reasonable" in the practical implementation of the optimisation approach held in Paris, last February 2017. The objective of this workshop was to initiate a discussion on the foundations of the optimisation principle and to share experience feedback on its implementation in three areas: the nuclear sector (particularly for occupational exposures), the medical sector and some existing exposure situations (exposure to radon, management of radium contaminated sites and management of postaccident situations). The article summarizes the discussions and conclusions of the workshop. It appears that the practical implementation of optimisation as well as the evaluation of the protection level considered as "reasonable" varies according to the field of activities, and remain a challenge in all cases.
\end{abstract}

Keywords: reasonable / radiological protection / optimisation / ALARA

\section{Introduction}

The principle of optimisation of radiological protection, known as ALARA (as low as reasonably achievable), is now the cornerstone of the radiological protection system as defined by the International Commission on Radiological Protection (ICRP) in its Publication 103 (ICRP, 2007a, b). In practice, its implementation is based on the search for a level of protection judged to be "reasonable" for a given exposure situation, taking into account economic and societal factors. The demonstration that exposures are as low as reasonably achievable is sometimes not sufficiently objectified and rarely made visible. Beyond the development of procedures such as multi-criteria analysis or cost-benefit analysis based on the adoption of monetary reference values of the person-sievert, the experience of implementing the optimisation principle shows that the "search for reasonableness" and the associated negotiating processes could be further discussed and shared at

\footnotetext{
*Corresponding author: valerie.chambrette@sfrp.asso.fr
}

the national and international level with the various stakeholders.

In 2016, IRPA launched a survey among the radiation protection societies to gather views on the practical implementation of the radiological protection system. The results of this survey were presented for the first time at the International Congress of Radiation Protection in Cape Town in May 2016. The survey shows that the system is sometimes (even often?) complex and difficult to explain to nonspecialists. It also appeared that it is not always easy to identify the reasons behind the adoption of a decision on protective measures. This calls for a greater visibility of the decision-making processes on the notion of "reasonable". Finally, a permanent question was reported: "How low is low enough?".

In this context, the French Society for Radiological Protection (SFRP) organised a workshop on the search for a level of protection judged to be "reasonable" in the practical implementation of the optimisation approach held in Paris, last February 2017. The objective of this workshop was to initiate a discussion on the foundations of the optimisation principle and 
to share experience feedback on its implementation in three areas: the nuclear sector (particularly for occupational exposures), the medical sector and some existing exposure situations (exposure to radon, management of radium contaminated sites and management of post-accident situations).

This workshop gathered 30 participants from radiological protection societies and international organisations. This paper summarizes the discussions and conclusions. The programme and presentations of the workshop are available on the SFRP website (Workshop presentations, 2017).

\section{The principle of optimisation and the issues associated with its implementation}

Introduced in the 1970s in ICRP Publications 22 and 26 (ICRP, 1973, 1977), the optimisation principle was initially based on the use of a cost-benefit analysis to judge the "reasonable" nature of protection actions: "...a reduction in collective dose can be compared directly with the detriment or cost of achieving this reduction." (ICRP 1973, sect. 18). This led notably to the adoption of tools such as reference monetary values of the person-sievert, particularly used in the nuclear industry for occupational exposures, but also for the management of public exposures.

Since cost-benefit analysis doesn't capture the various factors involved in the decision-making process, multi-criteria approaches were developed and the model of risk tolerability was adopted in the ICRP recommendations in the early $1990 \mathrm{~s}$ (ICRP, 1991). This model, applicable to practices (now the planned exposure situations), determines areas in which the exposure situation is deemed to be tolerable or unacceptable depending on the level of individual exposure and the efforts granted in order to achieve a level of risk reasonably "acceptable". In this context, stakeholder involvement has gradually emerged as a key issue in the decision-making processes.

In order to judge the reasonableness of an exposure situation, ICRP further described the optimisation approach in its Publication 101 by introducing a set of criteria taking into account economic, ethical and societal considerations and by promoting the stakeholder involvement process for implementing the evaluation and decision-making processes (ICRP, 2006). Publication 103, by confirming the optimisation principle as being the cornerstone of the radiological protection system, has also strengthened the role of stakeholder involvement in the search for "reasonableness" (ICRP, 2007a, b).

More recently, the ICRP's reflections on the ethical foundations of the radiological protection system led to the conclusion that "applying the principles of radiological protection (justification, optimisation and limitation) is a permanent quest for decisions that do more good than harm (beneficence/non-maleficence), that avoid unnecessary risk (prudence), that establish a fair distribution of exposures (justice) and treat people with respect (dignity)" (ICRP, 2017). The optimisation principle is seen, on the one hand, as " $a$ principle of action, which allows the practical implementation of prudence". On the other hand, "it also allows the introduction of equity, or fairness in the distribution of exposures among people exposed which refers directly to the ethical value of justice. Ultimately, taking into consideration the particular circumstances in which people are exposed as well as their concerns and expectations, the principle of optimisation respects people and treats them with dignity". Finally, it is interesting to note that the glossary of this document proposes a definition of "Reasonableness": "To make rational, informed and impartial decisions that respect other views, goals, and conflicting interests".

\section{ALARA in the nuclear sector}

In the nuclear sector, there has been a significant reduction of the occupational collective dose as well as a decrease of individual exposures since ICRP Publication 60 (ICRP, 1991). These results are mainly due to a structured implementation of the ALARA principle, the use of individual dose constraints in the optimisation process and a large contribution of feedback experience analysis to guide protection actions. However, it has to be stressed that while the mean individual occupational dose is rather low, there is a variable dose distribution depending on the specialty of the workers, which requires particular vigilance in the implementation of the optimisation process. In this sector, the use of cost-benefit analysis has been developed in the 1980s and 1990s as a decision-aiding tool for the selection of protection actions, especially when major investments have to be considered, usually associated with multi-criteria analysis for the final decision.

However, in an economic environment evolving to more difficulties, the continuation of the implementation of the ALARA approach for occupational exposures raises questions, such as:

- what are the optimisation margins for occupational exposures in normal operation of installations?

- how to implement a holistic/multi-risk and graded approach?

- what criteria should be used in assessing the reasonableness of protection investments, taking equity consideration into account?

- how to take into account potential risk transfer between workers (from different specialties) or between public and workers (when managing effluents, for example)?

These various questions show that the search for "reasonableness" is still under discussion. In particular, it appears that the point of view of operators and regulators may vary on this topic and it would be necessary to reinforce the dialogue between these actors in order to set and share the evaluation criteria used in the implementation of the optimisation process.

The specific issue of radioactive discharges management was also raised by the workshop participants: in most situations, it seems that the objectives of the implemented processes refer more to minimization than to optimisation. Furthermore, it appears also complex to take into account the political and environmental considerations adopted at both national and international levels. In this field, what is usually required in the use of the "best available technologies" and the question of the link between this approach and the search for "reasonableness" can be raised. 
Finally, the participants agreed that education and training as well as awareness-raising amongst stakeholders and the dissemination of the radiological protection culture must be strengthened in order to allow both effective protection and informed participation of the various stakeholders in the assessment and decision-making processes concerning protection measures in the nuclear sector.

\section{ALARA in the medical sector}

The primary goal of the use of radiation for medical purpose is to improve the quality of diagnostic and medical treatment. Optimisation of radiological protection applies to all exposed individuals: patients, staff, comforters and carers as well as members of the public not directly connected to the medical procedures.

In ICRP Publication 105, and the following publications dedicated to specific use of radiations in the medical field, ICRP underlines that for patient exposures, the expression "as low as reasonably achievable" (ALARA) is only part of the concept of optimisation and that the entire concept implies keeping patient exposure to the minimum necessary to achieve the required medical objective (diagnostic or therapeutic) (ICRP, 2007a, b, 2009, 2013; Bourguignon et al., 2017) That means that in some cases, patient doses need to be increased for the clinical outcome.

In practice, the ALARA approach in the medical field is driven by the specificities of medical practices (diagnostic/ interventional radiology, radiotherapy or nuclear medicine) in terms of level of individual exposures, relationship between patient and staff exposures as well as objectives of the use of radiation. In the discussion, the participants noted that the attention of the practitioners regarding patient exposure is initially focused on aspects of justification due to the highly importance of this principle in this sector, up to merging justification and optimisation principles.

The implementation of optimisation is a shared responsibility, involving not only the health professionals using radiation (surgeon, clinicians, radiologists...), the medical physicists or the radiographers, but also hospital managers who can play a key role to in setting the necessary organisation facilitating the implementation of the optimisation process taking into account budgetary constraints, and the equipment manufacturers.

The participants of the workshop underlined a difference in behaviour and/or radiological protection culture between, on the one hand, "prescribing" doctors, not always aware of radiological protection issues and, on the other hand, specialist doctors involved in diagnostic or therapeutic acts, more aware/ trained in radiological protection. Concerning health workers who work directly with patients, they are sometimes unaware of trained to radiological protection.

With regard to patients, it has been noticed that their direct involvement in the optimisation process is not so easy, due notably to their lack of knowledge regarding radiological protection issues. In some few cases, patients are represented by patient associations, which are generally focused on medical incidents. However, the obligation laid down in the new European Directive to inform patients about the dose received should help to raise their awareness, the main concern being then to avoid increasing fear of radiations (European Commission, 2013). For this purpose, the development of information and dissemination of a radiological protection culture among patients, health professionals and families is a key issue. The participants noticed that, for health professionals, the sharing of experience through the implementation of internal audits are considered as effective vectors for spreading radiological protection culture.

Among the remaining questions to be further studied, the participants of the workshop highlighted the challenges facing new technologies. While some of them, notably in diagnostic/ interventional radiology, allow a reduction of patient/staff doses, others, in particular in radiotherapy, may give rise to new types of incidents/accidents. For all these new technologies, there is a need to involve the relevant stakeholders (from manufacturers to end-users) for a better control of their use in a view to optimise protection and avoid incidents. The reinforcement of radiological protection education and training in medicine should also contribute to enhance progress in the implementation of optimisation of radiological protection for both patients and staff (Begnozzi et al., 2014).

It can be noticed that, as in the nuclear sector, the coordination with regulators was highlighted as necessary to assess the implementation of ALARA.

Lastly, in relation to the forthcoming Publication of the ICRP on the ethical foundation of the radiological protection system, the participants initiated reflections on the links between some principles and procedures associated with the medical use of radiation (informed consent, justification, inequity in patient dose distributions, etc.) and some ethical considerations applied in radiological protection (beneficence/ non maleficence, prudence, justice, dignity) (ICRP, 2017). These should be further developed in the future.

\section{ALARA in existing exposure situations}

The workshop focussed on the following existing exposure situations: exposure to radon, management of radium contaminated sites and management of post-accident situations.

It was first stressed that these situations were characterized by the fact that there was little or no control or capacity to intervene on the source, that there was a need to characterize the exposure situation before deciding to act and that there was an ability to intervene on the transfer routes and on the exposure pathways and habits (ICRP, 2007a, b). Moreover, for these situations, the persons directly exposed often play a key role in the implementation of protection actions and sometimes in the decision-making processes.

Very often, the approach put more emphasis on quality of life than on exposure levels (especially in the case of radon exposure and post-accident management). Similarly, the important role of ethical and societal considerations in the management of these situations was emphasized.

In this context where exposures already exist before any decision on protection action is taken, the optimisation approach is an iterative and generally long process aimed at gradually improving the situation. There is an acknowledgment that reaching zero risk is generally not achievable (for example, for environmental decontamination programmes 
after an accident or reduction of radon concentration in dwellings). The question then arises: what exposure level or contamination level is considered reasonable or sufficiently low? Here again the question arises of the competition between optimisation and minimisation approaches.

The practical implementation of the optimisation approach depends largely on the prevailing circumstances and the local context. During the workshop, two issues were particularly discussed for the implementation of the optimisation approach in these situations: the role of the reference level and the importance of the stakeholder involvement process with the associated development of the radiological protection culture.

\subsection{Role of reference level}

The choice of the values adopted for the reference levels constitutes a real challenge (Raskob and Landman, 2013). The reference level is very often considered by the various stakeholders as a limit value, and people tend to focus on the $1 \mathrm{mSv} /$ year value for public exposure in existing exposure situations. Furthermore, the participants noted that the use of derived reference levels (e.g., expressed in terms of measurable concentration or in dose rate) often lead to an increase of conservatism.

It was also stressed that individuals should be treated equitably in both existing and planned exposure situations. However, these situations are quite different and there is still a need to elaborate guidelines and to propose some set of values to determine reference levels in existing exposure situations.

Since then, it seems important to promote the establishment of a deliberative process and to increase transparency in the choice of values and selection of protection actions. While it is not central in setting the reference levels, health risk calculation is a sensitive issue that needs to be addressed in a comprehensible manner and avoiding the use of too conservative exposure assessments that may induce bias in the dialogue with stakeholders.

\subsection{Stakeholder involvement and radiological protection culture}

The experiences discussed during the workshop show that members of the public have difficulties in understanding the radiological protection system, the meaning of reference levels, and more generally the health risks associated with lowdose exposures. This reinforces the need to develop the radiological protection culture among the different stakeholders with a focus on actions and the specific context at the local level. In this perspective, local professionals, such as health professionals, education staff, and construction professionals..., have a key role to play. Finally, the importance of developing the conditions for social trust, in particular through enhanced transparency of decision-making processes and stakeholder involvement, was stressed.

As for the other sectors and perhaps more significantly, the multi-risk approach seems to be predominant in existing exposure situations, with the need for developing an integrated and graded approach tailored to each specific situation. At this regard, while it seems useful to put the radiological risk into perspective with other risks, this should be done carefully to avoid unsuitable comparisons. Finally, the question of the risk transfer between categories of workers, between workers and members of the public, between different populations or between generations remains to be further addressed.

\section{Conclusions / perspectives}

This workshop confirmed that the practical implementation of optimisation as well as the evaluation of the protection level considered as "reasonable" varies according to the field of activities, and remain a challenge in all cases.

Besides addressing specific exposure situations (such as radioactive waste management, public exposure associated with discharges of nuclear installations or hospitals, emergency exposures, ALARA in non-ionising radiation...), the perspectives identified for the different exposure situations are as follows:

- continue the analysis of the criteria adopted to assess the reasonableness of the protection actions;

- share feedback experience on the identification of the characteristics of each exposure situation, the risk assessment approaches, the decision-making processes, etc.;

- initiate a reflection on the implementation and the role of ethical and societal considerations for the various exposure situations;

- analyse the modalities for the practical implementation of a "graded approach";

- further analyse the component and the role of the radiological protection culture as well as the modalities for its development among the stakeholders;

- deepen the feedback experience and draw recommendations on the organisation of dialogue in the identification of the reasonable level of protection between the different stakeholders (authorities, operators, experts, public, patients, workers, etc.).

\section{References}

Begnozzi L, Cantone MC, Longobardi B, Veronese I. 2014. Prospective approaches for risk analysis in modern radiotherapy: the Italian experience and the contribution of medical physicists, Radioprotection 49(1): 43-47.

Bourguignon M, Bérard P, Bertho JM, Farah J, Mercat C. 2017. Radioprotection: quel avenir?, Radioprotection 52(1): 13-20.

European Commission. 2013. Council Directive 2013/59/EURATOM of 5 December 2013 laying down basic safety standards for protection against the dangers arising from exposure to ionising radiation.

ICRP Publication 22. 1973. Implications of commission recommendations that doses be kept as low as readily achievable, Ann ICRP.

ICRP Publication 26. 1977. Recommendations of the ICRP, Ann. ICRP 1: 3.

ICRP Publication 60. 1991. 1990 Recommendations of the International Commission on Radiological Protection, Ann. ICRP 21: $1-3$.

ICRP Publication 101b. 2006. The optimisation of radiological protection-Broadening the process, Ann. ICRP 36: 3. 
ICRP Publication 103. 2007a. The 2007 Recommendations of the International Commission on Radiological Protection. Ann. ICRP 37: 2-4.

ICRP Publication 105. 2007b. Radiological protection in medicine, Ann. ICRP 37: 6.

ICRP Publication 112. 2009. Preventing accidental exposures from new external beam radiation therapy technologies. Ann. ICRP 39: 4.

ICRP Publication 121. 2013. Radiological protection in paediatric diagnostic and interventional radiology, Ann. ICRP 42, 2.
ICRP. 2017. Report for consultation: ethical foundations of the system of radiological protection, to be published.

Raskob W, Landman C. 2013. Methodological aspects of the implementation of the new ICRP recommendations, Radioprotection 48(5): S43-S47.

Workshop presentations (2017). Available from http://www.sfrp.asso. $\mathrm{fr} /$ manifestations/manifestations/irpa-workshop-on-reasonable ness-in-the-implementation-on-the-alara-principle-. html, 9,38,0,0,2823.

Cite this article as: Schneider T, Lecomte J-F, Schieber C, Andresz S, Chambrette V, Le Guen B, Vaillant L. 2017. Synthesis of reflections and conclusions of the SFRP-IRPA workshop on the reasonableness in the practical implementation of the ALARA principle. Radioprotection 52(4): 259-263 